\title{
La mediación en las defensorías universitarias: perspectiva jurídica
}

\author{
Eduardo Gamero Casado \\ Exdefensor de las Universidades de Huelva y Pablo de Olavide
}

En recuerdo de Montserrat Casas i Ametller

\section{Resumen}

Este texto se corresponde con la intervención de su autor en el Encuentro de la CEDU celebrado en Córdoba en octubre de 2016. Analiza el marco jurídico de la mediación en las defensorías universitarias, exponiendo sus requisitos y su régimen de aplicación. Destaca la necesidad de aprobar un reglamento que regule la figura (puede ser la normativa de la propia Defensoría Universitaria), así como la imposibilidad de que los procedimientos iniciados como mediación se transfiguren después en procedimientos de queja.

\section{El concepto (jurídico) de mediación}

La mediación es un mecanismo alternativo de resolución de conflictos (técnicamente conocidos como ADRs, por el acrónimo de su expresión en inglés, Alternative Dispute Resolution): es un cauce que puede seguirse para resolver una disputa o enfrentamiento en lugar de seguir el sistema ritual de recursos, ya sean administrativos o judiciales.

Su origen no es institucional: apareció como una herramienta social para la gestión de los conflictos al margen de los sistemas institucionalizados y dotados de reconocimiento jurídico expreso. Se trataba, por tanto, de una realidad metajurídica que contribuía, sin embargo, a resolver conflictos, y que resultaba además idónea en ciertos ámbitos como la familia o la escuela, sin que el procedimiento a seguir estuviera determinado en una norma y sin que los acuerdos alcanzados por las partes llegaran a recibir un reconocimiento formal por el mundo del Derecho. Con el tiempo, se percibió su utilidad y comenzó a organizarse también de manera institucional, im- 
plantándose servicios públicos de mediación en los ámbitos más adecuados para su despliegue. El éxito que cosecharon las experiencias de mediación tanto en el sector público como en el privado hizo ver la oportunidad de regularlos formalmente, a fin de brindar un marco jurídico claro acerca de la aplicación de esta técnica de resolución de conflictos, y, sobre todo, de conferir eficacia jurídica a los acuerdos alcanzados por su medio. De esta manera, se abrió la posibilidad de utilizar la mediación como un instrumento rodeado de todas las garantías y plenitud de efectos jurídicos para resolver problemas que no solo podrían gestionarse así mediante un cauce más adecuado a la naturaleza del conflicto, sino que además permitía descongestionar los medios tradicionales y ritualizados de resolución de disputas. Por tanto, con la juridificación de la mediación se buscaban dos objetivos de carácter complementario: la reducción o aminoración de la litigiosidad formal; y la mejora de la eficacia en la resolución de conflictos, tanto en lo que se refiere al procedimiento (más ágil), como a sus resultados (más efectivo).

Los principales rasgos característicos de la mediación en el ámbito jurídico son al mismo tiempo los que definen a esta figura en el campo psicosocial. Contribuyen a conferirle un perfil específico y diferente de otros medios alternativos de resolución de conflictos. Se trata, concretamente, de los siguientes:

- Carácter autocompositivo: las propias partes buscan el acuerdo (aunque asistidas por un mediador). En cambio, en los métodos heterocompositivos (como el arbitraje) las partes se someten a los designios de un tercero, cuya autoridad aceptan, quien emite una posición vinculante para las partes en conflicto.

- Las partes conservan en todo momento el dominio del proceso, que pueden abandonar libremente.

- El mediador es un tercero imparcial (neutral), vinculado en todo momento (durante y después del proceso) por un deber de confidencialidad, y carente de poderes coactivos o de fuerza de obligar. Al mismo tiempo, debe ser alguien respetado por las partes, que le aceptan o suponen una cierta autoridad moral (auctoritas) y confían en su desempeño. Actúa como un mero posibilitador o catalizador del acuerdo entre las partes. Ahora bien, en función de cómo se haya configurado normativamente su figura, puede llegar a ser notario o fedatario del acuerdo, dejando constancia formal del mismo para acreditar fehacientemente su contenido y que las partes, llegado el caso, lo puedan hacer valer; así como garante de su cumplimiento. 
Aun cuando la mediación presenta estos caracteres homogéneos que la deslindan de otras figuras, también acoge en su seno una amplia variedad de procedimientos. En efecto, diversos aspectos del procedimiento se pueden adaptar a los deseos de las partes, de manera que pueden hacerse entrevistas sucesivamente con las partes o de manera simultánea (aunque esta segunda opción es más deseable, a veces resulta impracticable cuando el nivel de tensión es muy alto); las sesiones de mediación pueden ser más cortas o más largas; celebrarse en muy poco espacio de tiempo o dejando entre sí períodos más amplios, de reflexión; pueden tener un "guion" predeterminado o ir avanzando en los asuntos de manera más intuitiva, bajo los auspicios del mediador; puede levantarse acta de las sesiones o no; y así sucesivamente.

\section{Delimitación (jurídica) de la mediación respecto a otras figuras}

Para centrar adecuadamente el objeto de análisis es imprescindible diferenciar la mediación de otras figuras con las que presenta cierta similitud. La importancia de esta precisión se debe a la necesidad de no confundir las técnicas y procedimientos, en tanto que tienen distintos efectos legales. Conviene empezar por los buenos oficios, que es una gestión que alguien hace en beneficio de la posición de un tercero, auspiciando una causa que considera justa. Es propia del mundo diplomático, cuando se desata un conflicto entre dos partes y una de ellas se sirve de un tercero, dotado de cierta ascendencia o autoridad moral sobre la otra parte, para que defienda ante ella su posición. Es, también, la más frecuente de las actitudes de los defensores universitarios en los procedimientos que tramitan en sus oficinas, ya sea bajo la etiqueta de "consulta", "queja", o cualquier otra. De tal manera que, incluso la resolución de procedimientos ritualizados en las defensorías universitarias, mediante documentos escritos en los que se contienen sugerencias o recomendaciones, son encuadrables en esta categoría cuando pretenden suscitar la anuencia de la autoridad académica concernida respecto a la solución que se propone por el Defensor en beneficio del miembro de la comunidad universitaria promotor de la queja, toda vez que el Defensor carece de poderes ejecutivos y se limita a desplegar sus buenos oficios, respaldados por su auctoritas (moral), para postular una solución al conflicto. Conviene tener esto bien presente, pues a menudo los defensores piensan que cuando despliegan buenos oficios están mediando, e incluso a veces llegan a reflejarlo así en sus Memorias anuales cuando categorizan los asuntos en tipologías. Ciertamente, la diversidad de la carga semántica que corresponde al término "mediador" se presta a esta confusión, pero en el desempeño de nuestra labor debemos esforzarnos por evitarlo, pues la mayoría de defensorías universitarias tienen implantados procedimientos 
de mediación stricto sensu, y englobar en esta categoría los asuntos en que simplemente se han desplegado buenos oficios desvirtúa las estadísticas.

Por su parte, la negociación es también un remedio heterocompositivo para resolución de conflictos, y presenta muchas similitudes con la mediación, pero también la esencial diferencia de que, en la negociación, las partes no se encuentran gestionadas por un tercero, sino que pueden actuar por sí mismas o bien asistidas o representadas por otro (un experto o un simple interlocutor). La negociación es estrictamente bilateral, en tanto que la mediación es un procedimiento triangular. Y en la negociación, $a$ priori, cada parte busca la solución más ventajosa, aunque sea a costa del contrario y forzándole a ceder, en tanto que la mediación supone un escenario diferente, en el que, en línea de principio, las partes buscan proactivamente un mutuo entendimiento que se traduzca en una solución final ganar-ganar. Debido a estos perfiles, las defensorías universitarias no tramitan tales procedimientos de negociación.

Tenemos también la conciliación, que es una figura estrictamente procesal. Se trata, de hecho y en la mayoría de los casos, de un trámite previo a la formalización judicial del conflicto, y mediante el que se pretende evitar el proceso. Su más amplio espacio de operación es el Derecho laboral, en el que se articula un acto previo de conciliación, en presencia del juez, pero sin haberse iniciado propiamente el proceso, para ver si las partes alcanzan un acuerdo, que en tal caso quedaría rubricado y gozaría de la fuerza de cosa juzgada, evitándose el proceso, pero beneficiándose de sus mismos efectos.

Para concluir, el arbitraje es un procedimiento heterocompositivo de resolución de conflictos, en este caso no previo, sino alternativo al remedio judicial: las partes se someten voluntariamente al veredicto o laudo arbitral de un tercero, elegido de mutuo acuerdo por las partes (o mediante un sistema de designación que ambas partes han convenido previamente), y están obligadas a aceptar y avenirse al resultado del laudo, que recibe la fuerza de cosa juzgada y solo es susceptible de impugnación judicial en el caso de que se hayan infringido garantías del procedimiento (no por razones de fondo). Con los sistemas arbitrales se pretende, sobre todo, articular cauces rápidos de resolución de conflictos, más eficientes por ello que los judiciales, y también en el sentido de que los árbitros suelen ser especialistas en la materia sobre la que se pronuncian, en tanto que los jueces no siempre dominan el ámbito sobre el que les toca juzgar (pensemos en conflictos del sector eléctrico, inversiones internacionales, etc.). 


\section{3. Ámbitos de la mediación: pública o privada; su respectivo régimen jurídico}

Las técnicas de mediación que se aplican en ámbitos estrictamente privados y sin intención de recibir respaldo jurídico (o institucional) alguno, no precisan de un marco normativo de referencia. Evidentemente, no es la perspectiva que interesa aquí. Cuando se pretende conferir eficacia jurídica a la mediación el marco jurídico de aplicación es distinto según se trate de relaciones de Derecho público o de relaciones de Derecho privado. En este punto analizamos la medida en que estas normas pueden condicionar la mediación en las Defensorías Universitarias.

Comenzando por estas últimas, la mediación dispone, en primer lugar, de una legislación básica estatal, establecida en la Ley 5/2012, de mediación en asuntos civiles y mercantiles (LMACM). Su artículo 1 dispone: "Se entiende por mediación aquel medio de solución de controversias, cualquiera que sea su denominación, en que dos o más partes intentan voluntariamente alcanzar por sí mismas un acuerdo con la intervención de un mediador". Excluye de su ámbito de aplicación tanto la mediación laboral como la mediación con las Administraciones públicas. Por otra parte, en ejercicio de sus competencias en materia de servicios sociales, muchas Comunidades Autónomas han dictado normas en materia de mediación, como es el caso de la Ley 1/2009 de mediación familiar de Andalucía (LMFCAA). En cualquier caso, y por lo que se refiere a su aplicación a las universidades privadas, no parece que estas disposiciones impidan que estas instituciones articulen un mecanismo alternativo de resolución de conflictos entre los miembros de su comunidad universitaria, o entre estos y la propia Universidad, siempre que se haya establecido con la suficiente seguridad jurídica (mediante acuerdos de los órganos de gobierno competentes) y no cercene o limite el derecho a la tutela judicial efectiva de los miembros de la comunidad universitaria; el hecho de que la mediación sea por naturaleza voluntaria en todas sus fases, incluso en la firma del acuerdo, evita ese riesgo.

Por lo que se refiere a la mediación en el ámbito de las administraciones públicas (y por ende, de las universidades públicas), debemos distinguir a su vez entre conflictos que enfrenten a la institución con uno de los miembros de la comunidad universitaria (por ejemplo, cuando se dictan actos relativos a matriculaciones, becas, etc.); o los conflictos interpersonales que pueda llegar a haber entre miembros de la comunidad universitaria (por ejemplo, por enfrentamientos entre estudiantes, entre personal docente e investigador, personal de administración y servicios, o de integrantes de estos grupos entre sí): si estos últimos se mueven en el marco de las relaciones personales, y no desean trasladarse al plano disciplinario (o 
no tienen entidad para ello), se relajan bastante las garantías jurídicas que deben articularse para poderse desarrollar.

En cualquier caso, como el consejo que lanzamos desde estas páginas es el de regular la mediación en un reglamento interno (por ejemplo, el de la propia Defensoría Universitaria), debido a las limitaciones que pesan sobre la mediación en el ámbito de las relaciones de Derecho público, bastaría con seguir ese consejo para que dicho reglamento pueda aplicarse a todo tipo de relaciones (públicas y privadas), y solventar con ello las necesarias garantías legales.

Por lo que se refiere a la mediación en el marco de las relaciones de Derecho público, la figura se encuentra expresamente prevista en la Ley 39/2015, de procedimiento administrativo común de las administraciones públicas (LPAC), aunque de manera parca y poco estimulante para contribuir a su aplicación práctica. Esta Ley diferencia entre la posibilidad de aplicar la mediación i) En la vía de gestión, es decir, cuando se trate de adoptar una primera decisión en un asunto; o ii) En la vía de recurso, esto es, una vez que se ha tomado una decisión (acto administrativo) y procede impugnarlo en vía administrativa (recursos de alzada o reposición, principalmente).

Por lo que se refiere a la vía de gestión, el artículo 86 de la LPAC, bajo la rúbrica "terminación convencional", permite que, antes de adoptarse la decisión (el acto administrativo mediante el que se resuelve el procedimiento), se desarrolle una fase de mediación para que las partes puedan llegar a un acuerdo. La Ley establece tres requisitos principales para que puedan suscribirse estos acuerdos. El primero es que se apruebe previamente una disposición que los regule. En el caso de las universidades, puede ser un reglamento; y ese reglamento puede ser, evidentemente, el que regule la figura del Defensor Universitario. Se requiere, en segundo lugar, que no versen sobre materias no susceptibles de transacción y tengan por objeto satisfacer el interés público que tienen encomendado. Esto hace inviable que se plantee la mediación en supuestos de aplicación del régimen disciplinario, ya sean estudiantes o empleados públicos, pues se trata de una materia de orden público y, en cuanto que tal, indisponible. Ahora bien, lo que sí resulta perfectamente posible (y de hecho viene sucediendo en las defensorías), es que un miembro de la comunidad universitaria que ha sufrido una afrenta que podría calificarse como infracción disciplinaria (ya sea de un estudiante, ya de un PAS o de un profesor), acuda a la defensoría universitaria solicitando la mediación de la oficina para obtener mediante ella una reparación moral, evitando de esa manera presentar una denuncia a las autoridades académicas y renunciando por consiguiente a la incoación de un expediente disciplinario. Por tanto, se puede mediar antes, pero no durante la tramitación de un expediente disciplinario.

El tercer requisito establecido por la LPAC es el de que los acuerdos al- 
canzados establecerán como contenido mínimo la identificación de las partes intervinientes, el ámbito personal, funcional y territorial, y el plazo de vigencia, debiendo publicarse o no según su naturaleza y las personas a las que estuvieran destinados. Se debe tener presente que la norma puede aplicarse a cualesquiera conflictos de las administraciones públicas, y de ahí algunas de las previsiones de este precepto, en el que se adivina cierta propensión a la resolución por vía de mediación de conflictos colectivos en materia de empleo público. Lo normal en las defensorías universitarias es que no sea necesario precisar más ámbito que el de los propios intervinientes; que no haya verdaderamente un "plazo de vigencia", sino un seguimiento posterior del cumplimiento del acuerdo; y que este no deba publicarse, pues sus efectos se circunscribirán a las propias partes intervinientes.

En cuanto a la vía de recurso, el artículo 112.2 de la LPAC permite sustituir el recurso de alzada y el de reposición por procedimientos de mediación, sujetando esta posibilidad al cumplimiento de tres condiciones. La primera es que lo justifique la especificidad de la materia. Parece que las peculiaridades de las relaciones que se entablan en la Universidad permitirían cumplir esta exigencia. La segunda condición es que se establezca en una norma con rango de ley. Dado que no existe ninguna ley que regule la mediación en las universidades, esta previsión legal, que también existía en la anterior Ley 30/1992 (LRJAPPAC), impediría a las universidades implantar la mediación en vía de recurso. Ahora bien, la Ley de procedimiento de 2015 plantea una novedad que supone una vía de solución a este problema: el hecho de que establece que las universidades:

Se regirán por su normativa específica en el ejercicio de las funciones públicas que les hayan sido atribuidas por Ley o delegadas por una Administración Pública, y supletoriamente por la presente Ley (art. 2.2.c LPACAP).

Los posicionamientos doctrinales que se han producido acerca de esta nueva previsión legal, entre los que yo mismo me encuentro, han rechazado la posibilidad de que un mero reglamento universitario conculque $o$ limite las garantías que tienen todos los ciudadanos en el procedimiento administrativo común. Pero en la materia en examen la cuestión es distinta: se trata, precisamente, de que la normativa específica de la Universidad aplique los instrumentos que la propia Ley diseña, adoptándolos con el rango normativo que le cabe a su propia capacidad jurídica, es decir, el reglamentario. Por consiguiente, entiendo que, en aplicación del artículo 2.2.c) de la LPAC, las universidades pueden aprobar reglamentos que reúnan los requisitos del artículo 112.2 de la propia Ley para regular la mediación en 
sustitución de los recursos de alzada o reposición respecto de los actos que dicte la propia Universidad.

Por último, la tercera condición es que el procedimiento tenga lugar ante órganos colegiados o comisiones específicas no sometidas a instrucciones jerárquicas. El contenido esencial de este precepto es la independencia del mediador, más que el carácter colegiado del órgano. No parece por tanto que ello suponga una objeción real a la implantación de la mediación en las defensorías. En cualquier caso, para despejar definitivamente puede aplicarse sin violencia la misma consideración que hemos apuntado en el requisito anterior sobre la supletoriedad de la LPAC en el ámbito universitario.

\section{El marco jurídico de la mediación en la Universidad}

La vertiente organizativa es esencial en los medios extrajudiciales de resolución de conflictos del sector público (Tornos, 1995, 156 y ss.). La funcionalidad de cada modelo depende esencialmente de la tipología del conflicto y del tamaño de la administración u organización implicada. Por un lado, no cabe mediar en todos los conflictos, como ya hemos visto; por el otro, cuanto mayor sea el tamaño de la institución, más complicado puede ser articular un sistema de mediación susceptible de llegar a todos los potenciales interesados y capaz de gestionar adecuadamente todos los procedimientos.

Existen dos vías principales de implantación de la mediación en la Universidad: mediante servicios específicos de mediación, como los que ha creado, por ejemplo, la Universidad Complutense de Madrid; o mediante las Defensorías Universitarias, regulándolos en sus reglamentos orgánicos, con el amparo de la disposición adicional $14^{\mathrm{a}}$ de la Ley Orgánica 6/2001, de Universidades (LOU). Conceptualmente se trata de mediadores "institucionales" (Six, 1997, 154 y ss.) o "administrativos" (Aclanda, 1993, 294 y ss.), esto es, órganos especialmente concebidos para asumir esta función, lo cual los diferencia de los mediadores del ámbito privado.

Centrándonos en la figura de la Defensoría Universitaria, debemos notar, en primer lugar, la idoneidad del Defensor como mediador, en atención a sus rasgos institucionales, muchos de los cuales aparecen consignados en la propia LOU:

- Ausencia de poderes ejecutivos: no puede imponer su criterio.

- Modo de designación: autoridad moral, generación de confianza.

- Confidencialidad.

- Independencia. 
Pero además, y conforme hemos comprobado en el apartado anterior, se deben cumplir determinadas exigencias formales; en particular, la competencia de mediación y su concreto procedimiento de ejercicio deben estar expresamente regulados en la normativa universitaria (preferentemente, Reglamento o Normativa de la Defensoría Universitaria), para que no haya dudas de la titularidad de la competencia, y queden suficientemente regulados sus efectos jurídicos (en aras de la seguridad jurídica).

Además, ese reglamento debe prever que no se pueda pasar a tramitar como queja un asunto iniciado como mediación en el que no se llegue a acuerdos, pues lo contrario desnaturaliza la mediación como técnica alternativa de resolución de conflictos, y la convierte en otra cosa. Las partes deben saber por anticipado que, si acuden a la Defensoría solicitando mediación, e inician el procedimiento, en el caso de que este no concluya mediante acuerdo, el Defensor no puede transfigurarlo de oficio en un procedimiento de queja, y tampoco les cabe a las partes promover esa transustanciación. El motivo de fondo de esta previsión es material y no formal: en el escenario de confidencialidad que preside la mediación, las partes pueden confesar cosas que no dirían en un procedimiento heterocompositivo (como sería el procedimiento de queja, o en la instrucción de un recurso administrativo), de tal manera que, si las partes pudieran salirse del procedimiento y utilizar la mediación para otros fines, estarían de hecho abusando del procedimiento y desvirtuándolo, convirtiéndolo en un puro medio para obtener información privilegiada.

Son ya muchos los reglamentos de las defensorías universitarias que regulan este procedimiento y que pueden servir de inspiración para quienes deseen promover una norma de esta naturaleza en su propia Universidad. En la Universidad Pablo de Olavide, yo mismo propuse el texto de la Normativa de la Defensoría Universitaria, aprobado por el Consejo de Gobierno en 2010, que regula la mediación en sus artículos 20 y siguientes ${ }^{1}$.

A continuación podríamos detenernos en desarrollar las fases de la mediación, la forma de articular los acuerdos y de monitorizar su cumplimiento, pero se hace innecesario porque son cuestiones espléndidamente tratadas por otras fuentes más cualificadas, a las que asimismo presta atención el artículo de Carlos María Alcover, publicado en este mismo número, y sobre las que existe una actividad constante de información y formación por parte de la CEDU. Por todo ello, y por la moderada extensión que deben tener estas páginas, considero preferible no abordarlas aquí.

1 Aunque han pasado ya diez años y cambiaría algunas cosas, por si fuera de interés, el texto está disponible online (Reglamento UPO/CG/2010, de 20 de diciembre). 


\section{Dificultades específicas de la mediación en el ámbito público y universitario}

Existen varios aspectos que dificultan el despliegue de la mediación en el ámbito público y, especialmente, en el universitario. Tenemos, en primer lugar, la imposibilidad de transigir en materias de orden público, que ya hemos comentado con anterioridad. Por otro lado, los miembros de la comunidad universitaria presentan grandes desequilibrios de estatus, lo que dificulta que personas que se encuentran en niveles diferentes de jerarquía (un catedrático consagrado y un estudiante de primer curso o un becario de investigación) se sienten a la misma mesa en un plano de igualdad. La articulación de la mediación a través de las Defensorías es muy positiva, porque contribuye a igualarlas, a equilibrar la posición de las partes mediante la interposición de una figura neutral y dotada de autoridad moral. Pero lo cierto es que, a menudo, quien se ve en situación de primacía no encuentra aliciente a entrar en un procedimiento que le hace perder su posición y aventurarse a que se expongan relatos comprometedores. Por otra parte, incluso en el caso de que se avengan a participar en el procedimiento, cabe que lo abandonen cuando se sientan amenazados en su prestigio o consideración por el devenir del procedimiento, refugiándose de nuevo en su categoría y haciendo derivar el asunto hacia los cauces tradicionales (que el afectado presente una reclamación ante otras autoridades académicas).

Para concluir, también se presentan dificultades específicas en los conflictos de personal, especialmente en el personal docente e investigador, $\mathrm{y}$ todavía más acusadamente en universidades pequeñas o con campus único. Por una parte, la estructura de las enseñanzas universitarias es muy rígida, adscribe a los profesores a áreas de conocimiento en las que permanecen de por vida (académica), siendo así, además, que la promoción se configura habitualmente como una carrera horizontal, en la que el profesor permanece en el mismo sitio, pero va mejorando el nivel de su puesto de trabajo (ayudante-contratado doctor-titular-catedrático). Esto no solo conduce a que los conflictos se enquisten, sino que además no deja muchas salidas a la hora de encontrar soluciones: no solo será inviable un cambio de puesto, sino incluso de centro, para lograr que la distancia física permita a las partes distanciarse también emocionalmente del problema y reconstruir poco a poco su convivencia.

Todo esto se expone en una intervención relativa a los aspectos jurídicos de la mediación sencillamente para advertir que el Defensor Universitario debe ser consciente de las limitaciones legales que pesan sobre su labor: por una parte, evitando mediar en los asuntos que no cabe tramitar legalmente por este procedimiento; y por otra, teniendo presente los límites de los acuerdos a que puedan llegar las partes, que no pueden rebasar la rígida 
estructura establecida en el empleo público en general, y en el universitario en particular.

Por lo demás, la mediación se ha revelado como una valiosa herramienta para la resolución de conflictos en las Defensorías Universitarias. Encuentra su espacio idóneo, como ya se ha dicho, en relación con los conflictos interpersonales, en los que el régimen de la disciplina académica es obsoleto y no aporta soluciones que verdaderamente acaben con los conflictos. Tengo recuerdos muy especiales de algunos de estos procedimientos a lo largo del desempeño de la Defensoría en las Universidades de Huelva y Pablo de Olavide, con resultados emotivos a pesar de la complejidad de los asuntos abordados y la intensidad de las sesiones de mediación; en la mayoría de estos casos, se trataba de asuntos en el que excelentes relaciones personales previas (entre profesores, entre estudiantes, entre miembros del PAS, entre unos y otros...) se habían deteriorado hasta extremos lamentables, y el conflicto que inicialmente llegaba la defensoría no era más que un episodio puntual de esa convulsa relación: a través de las mediaciones se deshicieron muchos equívocos y malentendidos, las diferencias (las de fondo) se fueron diluyendo, y después de sesiones a menudo largas y con estallidos de ira, se acababa serenando el ambiente en una especie de catarsis liberadora que unía nuevamente a als personas. Pero, como contrapunto, y según lo que ya sabemos, insisto en la idea de que la mediación no es el bálsamo de fierabrás: no vale para todo, e incluso en algunos casos en los que es el mecanismo idóneo para resolver un conflicto, cabe la posibilidad de que, finalmente, no se logre un acuerdo entre las partes. Esto, por muy frustrante que sea, no desdice su utilidad, sino que más bien nos anima a redoblar los esfuerzos para que la cultura de la mediación se extienda en las sociedades contemporáneas; tanto más, en un caldo de cultivo tan idóneo como es la comunidad universitaria, a la que se le presume un talante abierto, proactivo y dialogante.

\section{La CEDU y la mediación}

Importa destacar el papel que viene desempeñando la CEDU para potenciar la mediación en las Defensorías Universitarias. Hasta donde alcanza mi memoria, la primera ocasión en que esta cuestión fue objeto de atención específica fue con ocasión del Encuentro de Palma de Mallorca, en 2004. Bajo los auspicios de Montserrat Casas (a cuyo admirado recuerdo se dedican estas líneas), se analizó la mediación en una mesa de trabajo que propuso al pleno un texto, que se adjunta como Anexo para facilitar su localización. En esa ocasión se giró consulta a las Defensorías Universitarias: de acuerdo con sus respuestas, dos tercios de las Defensorías realizan actividades de mediación, y cerca del $25 \%$ de los conflictos se resuelve por esta vía. 
Con posterioridad, la CEDU ha fomentado extraordinariamente el tratamiento de esta materia en otros muchos Encuentros (el de Córdoba, en el que se pronunció la intervención que está en el origen de este artículo, es un claro exponente de ello), y ha propiciado u organizado cursos formativos y actividades que permitan a los Defensores desarrollar las habilidades necesarias para el desempeño de esta delicada misión.

Celebro que las Defensorías sigan potenciando esta valiosa herramienta de resolución de conflictos que tanto contribuye a la consecución de fines y valores inherentes a la cultura universitaria. Como se sostiene en el párrafo final de la declaración de Palma de Mallorca que se transcribe a continuación:

Se trata de favorecer en toda la comunidad universitaria la generalización de una actitud conciliadora, responsable y mediadora, que considere el conflicto como algo positivo - funcional-, en tanto que contribuye al cambio y a la mejora de la calidad universitaria en todos los aspectos. 
Anexo: Texto final del documento de trabajo de la mesa 3, organizada durante el VII Encuentro Estatal de Defensores Universitarios (Baixeras y Delclos y Gamero Casado (coord.), 2004).

La mediación constituye una herramienta a disposición del Defensor Universitario para el cumplimiento del fin institucional de contribución a la mejora de la calidad universitaria en todos sus ámbitos que establece la disposición adicional $14^{\mathrm{a}}$ de la LOU.

La premisa básica sobre la que se funda la mediación es que lo importante para resolver los conflictos es conocer sus causas, para resolverlos en sus fundamentos y no en sus manifestaciones puntuales. Su otro componente esencial es que pretende que todas las partes implicadas encuentren por sí mismas un acuerdo que dirima su controversia, actuando el mediador como promotor o catalizador de dicho acuerdo. En este sentido, el procedimiento de mediación ha de ser voluntario en todos sus elementos: la propia aceptación de la mediación por las partes, fases que atravesará el procedimiento, y acuerdos a los que pueda llegarse.

El Defensor Universitario puede utilizar la mediación de dos maneras o vías diferentes:

- Mediación en sentido estricto, como procedimiento específico encuadrado entre los cauces alternativos de resolución de disputas cuya gestión se le confían, junto a las quejas, las consultas u otros.

- Mediación en sentido genérico, como técnica puntual aplicada intuitivamente, o de manera inespecífica, en una fase determinada de un asunto sometido a su consideración.

Un aspecto a debatir es la eventual existencia de límites éticos a la actividad de mediación del Defensor Universitario. En primer término, esta cuestión evoca la tipología de asuntos o materias insusceptibles de mediación en razón de su naturaleza; y en segundo lugar, plantea la necesidad de que el mediador no se enclaustre en un papel estimulador de cualesquiera acuerdos entre las partes, sino que vele por el respeto de unos límites éticos en los arreglos a que se llegue con ocasión de la mediación. Esto último invoca el delicado asunto de concretar dónde se situarían tales límites morales cuya custodia se confía al Defensor.

En principio, cabe la mediación en cualesquiera materias en las que sea posible vislumbrar una solución razonable, salvo en los siguientes supuestos:

- Cuando estén comprometidos derechos fundamentales o irrenunciables. 
- Cuando existan normas imperativas que no permitan dispensa o exención.

La extraordinaria variedad de asuntos que pueden presentarse ante la oficina del Defensor desaconseja la protocolización de la actividad de mediación mediante la aprobación de un procedimiento-tipo cuyas fases resulten de obligado seguimiento en todo proceso de mediación. No obstante, será útil para el Defensor Universitario conocer diferentes plantillas o modelos de procesos de mediación, que puedan ilustrarle y enriquecer sus aptitudes para la gestión de los conflictos.

No resulta preciso ni conveniente esclerotizar el procedimiento de mediación con un protocolo predeterminado. Ahora bien, el Defensor en funciones de mediación podrá confeccionar un procedimiento específico para cada caso, según los factores sustantivos (materia sobre la que verse) y personales (perfil de las partes) incidentes en el caso.

En el caso de que lo permita el régimen aplicable a cada Defensor Universitario, puede ser de gran ayuda que el Defensor en funciones de mediación acuerde expresamente ese procedimiento con las partes antes de iniciar la mediación propiamente dicha. Los aspectos a contemplar en ese procedimiento de mediación "a medida" serían:

- Si hay reuniones conjuntas de las partes o presentación de posiciones por separado.

- Si el resultado de la mediación se plasma en un acuerdo escrito o no.

- Si ese acuerdo final se firma formalmente o no.

- Si el Defensor realizará un seguimiento de su ejecución o no.

También puede ser útil que el Defensor tenga a disposición de las partes una o varias plantillas de procesos de mediación para que consideren si alguno de ellos se adapta a su problema.

Para el desempeño de funciones de mediación por el Defensor Universitario es recomendable un conocimiento básico de técnicas psicológicas y sociológicas de gestión de conflictos, así como de habilidades para potenciar e interpretar la información facilitada por las partes, ya se trate de estímulos a la comunicación, ya la interpretación de información subliminal - por ejemplo, la comunicación no verbal-, ya a otras estrategias y pericias de utilidad para la mediación. Sería muy conveniente que, con ocasión de los encuentros de Defensores Universitarios, se atendiese a esta faceta formativa, ya sea mediante la impartición de conferencias, ya mediante talleres o seminarios específicos, que formen a los Defensores Universitarios en técnicas, tácticas y estrategias de mediación.

En definitiva, se trata de favorecer en toda la comunidad universitaria la 
generalización de una actitud conciliadora, responsable y mediadora, que considere el conflicto como algo positivo -funcional-, en tanto que contribuye al cambio y a la mejora de la calidad universitaria en todos los aspectos.

\section{Legislación citada}

LMACM

Ley 5/2012, de 6 de julio, de Mediación en Asuntos Civiles y Mercantiles. Boletín Oficial del Estado, núm. 162, 7 de julio de 2012, pp. 49224-49242.

\section{LMFCAA}

Ley 1/2009, de 27 de febrero, reguladora de la Mediación Familiar en la Comunidad Autónoma de Andalucía. Boletín Oficial del Estado, núm. 80, 2 de abril de 2009, pp. 3127431287.

LOU

Ley Orgánica 6/2001, de 21 de diciembre, de Universidades. Boletín Oficial del Estado, núm. 307, 24 de diciembre de 2001, pp. 49400-49425.

LPACAP

Ley 39/2015, de 1 de octubre, del Procedimiento Administrativo Común de las Administraciones Públicas. Boletín Oficial del Estado, núm. 236, de 2 de octubre de 2015, pp. 8934389410.

\section{LRJAPPAC}

Ley 30/1992, de 26 de noviembre, de Régimen Jurídico de las Administraciones Públicas y del Procedimiento Administrativo Común. [Disposición derogada]. Boletín Oficial del Estado, núm. 285, de 27 de noviembre de 1992, pp. 40300-40319.

Reglamento UPO/CG/2010, DE 20 DE DiCiEMBRE

Reglamento de la Defensoría Universitaria de la Universidad Pablo Olavide. Disponible online: https://www.upo.es/du/normativa/ (último acceso: 8 de diciembre de 2017).

\section{Bibliografía citada}

ACLAND, ANDRew Floyer. (1993). Cómo utilizar la mediación para resolver conflictos en las organizaciones. Barcelona: Ediciones Paidós.

Baixeras y Delclos, Isabel y Gamero Casado, Eduardo (coord.). (2004). "Mesa de trabajo no 3. La actividad de mediación del Defensor Universitario". Documento de conclusiones realizado durante el VII Encuentro Estatal de Defensores Universitarios, Universidad de 
las Islas Baleares, 26-28 de octubre de 2004. Disponible online: http://www.cedu.es/ images/encuentros/estatales/VIIEncuentro/07.conclusiones3.pdf (último acceso: 8 de diciembre de 2017).

Six, JEAn-François. (1997). Dinámica de la mediación. Barcelona: Paidós.

Tornos Mas, Joaquín. (1995). "Medios complementarios a la resolución jurisdiccional de los conflictos administrativos", Revista de Administración Pública, 136, 149-178. 